\title{
Closed-Form Design of Maximally Flat FIR Fractional Delay Filters
}

\author{
Soo-Chang Pei and Huei-Shan Lin \\ Department of Electrical Engineering \\ National Taiwan University \\ Taipei, Taiwan 10617, ROC \\ pei@cc.ee.ntu.edu.tw
}

\author{
Peng-Hua Wang \\ Department of Electrical Engineering \\ Chang Gung University \\ Taipei, Taiwan 333, ROC \\ phwang@mail.cgu.edu.tw
}

\begin{abstract}
FIR fractional delay filters are proposed. They are designed by separately approximating the real part and the imaginary part of the frequency response. Among these 8 types of filters, two of them are equivalent to the existing design results in the open literature [6][9]. Therefore, a more general closed-form design of the maximally flat FIR fractional delay filters is described in this paper and some design examples of FIR fractional delay filters will be illustrated.
\end{abstract}

\section{INTRODUCTION}

Application and design of digital fractional delay (FD) filters have been widely studied in the open literature [1] Standard techniques such as windowing method, equiripple, or maximally flat approximation can be applied to designing FD filters [1][2]. In [1], the authors provide a comprehensive review about the FD filter performance of several design techniques.

Maximally flat (MF) design of FD filters attracts many investigations because of its close relation to the theory of interpolation [1][4][5][6]. Several results on the closed-form coefficients are derived [1] [3][6] [8]. These closed-form expressions are explicitly solved from linear equations derived from the MF conditions. It is interesting that the coefficients can be also derived from power series expansion of suitable basic functions [6][7]

In this article, we propose several types of finite impulse response (FIR) filters for approximating FD systems. The impulse response of each type of filters are solved by MF approximation and expressed in closed form. Properties of each type of filters are investigated and lead to some conclusion on its performance.

\section{FORMULATION}

The $N$ th order FIR filter with its impulse response $\left\{h_{n} \mid n=0,1, \ldots, N\right\}$ can be expressed by

$$
H(z)=h_{0}+h_{1} z^{-1}+\cdots+h_{N} z^{-N}=\sum_{n=0}^{N} h_{n} z^{-n}
$$

whose frequency response is represented as

$$
H\left(e^{j \omega}\right)=h_{0}+h_{1} e^{-j \omega}+\cdots+h_{N} e^{-j N \omega}=\sum_{n=0}^{N} h_{n} e^{-j n \omega}
$$

The fractional delay filter with desired delay $D$ has the ideal frequency response of

$$
H_{\text {ideal }}\left(e^{j \omega}\right)=e^{-j D \omega} .
$$

We can write $D$ as $D=K+d$ or $D=K+1 / 2+d$ and obtain the fractional part $d$ of the desired delay where $K$ is an integer and $0<d<1$. Based on the separation of integer part and fractional part, the desired frequency response can be written as

or

$$
H_{\text {ideal }}\left(e^{j \omega}\right)=e^{-j D \omega}=e^{-j K \omega} \cdot e^{-j d \omega}
$$

$$
H_{i d e a l}\left(e^{j \omega}\right)=e^{-j D \omega}=e^{-j(K+1 / 2) \omega} \cdot e^{-j d \omega} .
$$

Accordingly, we will use

$$
H_{d}\left(e^{j \omega}\right)=e^{-j d \omega}
$$

as the desired frequency response in this article.

\section{DESIGN OF MF FIR FD FILTERS}

In this section, we propose 8 types of FIR filters to implementing FD system based on the symmetry of the desired frequency response in (4), and solve its coefficients by MF approximation. The desired frequency response can be written as $H_{d}\left(e^{j \omega}\right)=\cos (d \omega)-j \sin (d \omega)$ where the real part is an even function and the imaginary part is an odd function. A natural way to implement functions with such symmetry by digital filters is to express the even function as cosine series and to express the odd function by sine series. Let $H(\omega)$ be the target frequency response. Formally, the cosine series and the sine series are expressed as $\cos ((P i+a) \omega)$ and $\sin ((P i+b) \omega)$ where $P \in\{1,2\}$ and $a, b \in\{0,0.5\}$ in the case of $P=1, a, b \in\{0,1\}$ in the case of $P=2$. Therefore, we obtain 8 ways to represent $H(\omega)$ as an approximation of the desired frequency response. In this

This work is supported by the National Science Council, R. O. C., under Contracts NSC93-2219-E-002-004 and NSC93-2752-E-002-006PAE. 
article, we will focus on the derivation of the same number of coefficients on the approximation of real part and imaginary part. The 8 proposed frequency responses and associated transfer functions are listed in Table 1. Moreover, the impulse responses for the case $M=5$ are depicted in this table to illustrate their behavior of symmetry.

table I. The Proposed Frequency Responses And The Associated Causal Transfer Functions

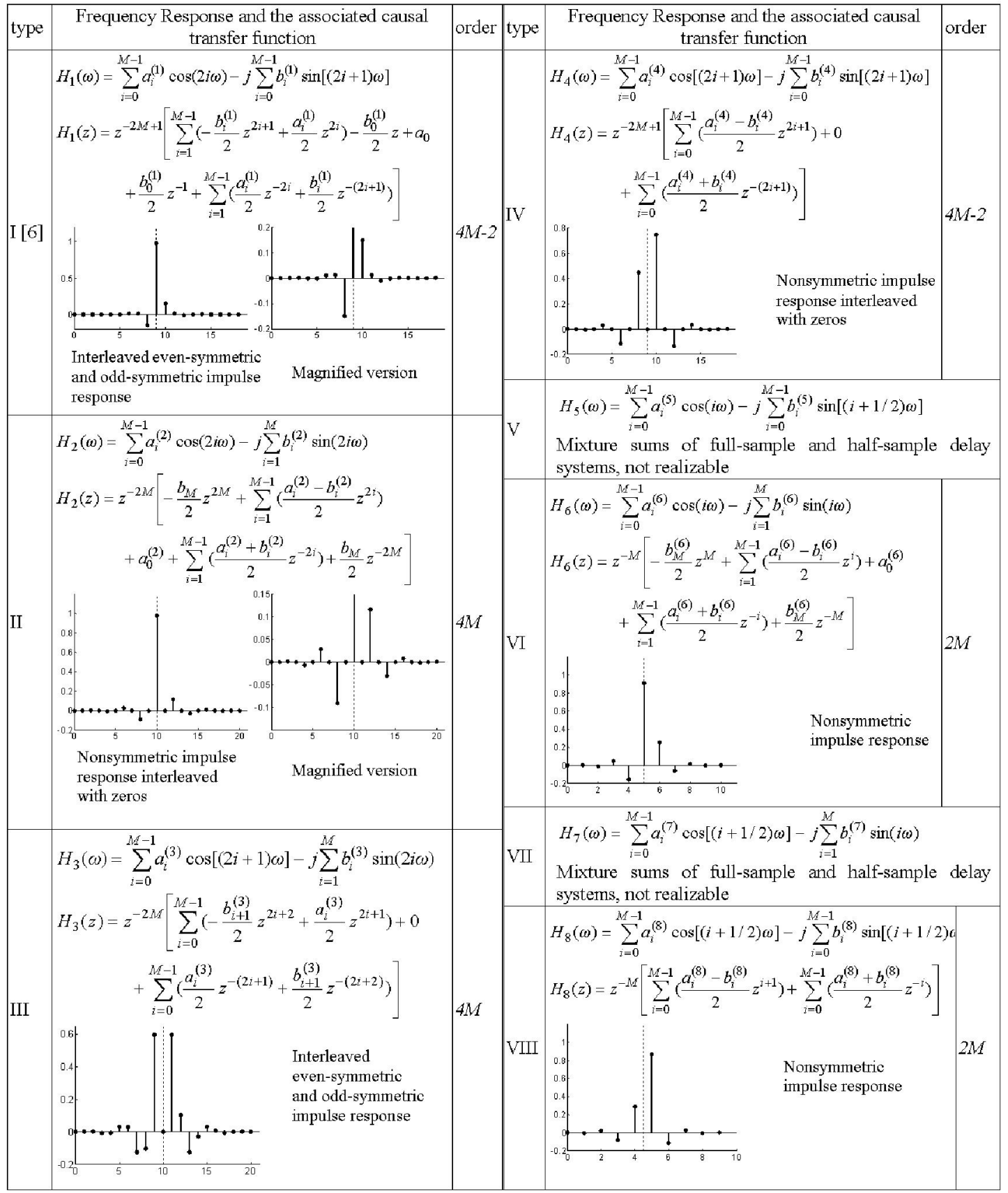

We make a simple discussion before designing filters having the frequency responses in Table 1. All the 8 types of frequency responses are the sums of two linear-phase systems. One contributes the real part and another contributes the imaginary part. Type I filter is equivalent to the FIR FD filter proposed in section IV of [6]. Frequency 
responses of type-V and VII are sums of a full-sample and a half-sample delay systems where the complete design of filters with this mixture is not realizable in practice. The transfer function of type-VIII is just another expression of the general transfer function in (1) for $N=2 M-1$. However, the transfer function of type-VI is not identical to the general transfer function in (1) for $N=2 M$. A simple observation about the fact is that the last impulse response of $z^{-2 M}$ is negative to the constant term in $H_{6}(z)$. But the impulse response of the general transfer function does not be limited by this constraint. However, the filters of type VI and VIII are exactly equivalent to the filters proposed in [9].

In the following, we will derive the coefficients of the filters listed in Table 1 . Let $\left\{A_{i}(d) \mid i=0,1, \ldots, M-1\right\} \quad, \quad\left\{B_{i}(d) \mid i=0,1, \ldots, M-1\right\}$ and $\left\{C_{i}(d) \mid i=1,2, \ldots, M\right\}$ be the solutions of the following 3 set of linear equations:

$$
\begin{aligned}
& \sum_{i=0}^{M-1} A_{i}(d)(2 i)^{2^{2}}=d^{2 p} ; \quad p=0,1, \ldots, M-1, \\
& \sum_{i=0}^{M-1} B_{i}(d)(2 i+1)^{2 p}=d^{2_{p}} ; \quad p=0,1, \ldots, M-1, \\
& \sum_{i=1}^{M} C_{i}(d)(2 i)^{2 p}=d^{2_{p}} ; \quad p=0,1, \ldots, M-1,
\end{aligned}
$$

respectively. We will show that all the coefficients of the 8 types of filters can be expressed by these common factors $A_{i}(d), B_{i}(d)$ and $C_{i}(d)$. We first solve the above 3 sets of linear equations by Cramer's rule. The closed-form solutions are

$$
\begin{gathered}
A_{0}(d)=\frac{(-1)^{M-1}\left(d^{2}-2^{2}\right)\left(d^{2}-4^{2}\right) \cdots\left[d^{2}-(2 M-2)^{2}\right]}{[(M-1) !]^{2} \times 2^{2 M-2}}, \\
A_{i}(d)=\frac{2(-1)^{M-1-i}\left(d^{2}-0^{2}\right)\left(d^{2}-2^{2}\right) \cdots\left[d^{2}-(2 M-2)^{2}\right]}{\left[d^{2}-(2 i)^{2}\right] \times(M-1-i) ! \times(M-1+i) ! \times 2^{2 M-2}}, \\
\quad i=1,2, \ldots, M-1, \\
B_{i}(d)=\frac{(4 i+2)(-1)^{M-1-i}\left(d^{2}-1^{2}\right)\left(d^{2}-3^{2}\right) \cdots\left[d^{2}-(2 M-1)^{2}\right]}{\left[d^{2}-(2 i+1)^{2}\right] \times(M-1-i) ! \times(M+i) ! \times 2^{2 M-1}}, \\
\quad i=0,1, \ldots, M-1 \\
C_{i}(d)=\frac{8 i^{2}(-1)^{M-i}\left(d^{2}-2^{2}\right) \times\left(d^{2}-4^{2}\right) \times \cdots \times\left[d^{2}-(2 M)^{2}\right]}{\left[d^{2}-(2 i)^{2}\right] \times(M-i) ! \times(M+i) ! \times 2^{2 M}}, \\
i=1,2, \ldots, M
\end{gathered}
$$

To find the coefficients of $H_{1}(\omega)$, we set the MF condition at $\omega=0$ and obtain a set of linear equations consisting of $2 M$ equations

$$
\left.\frac{d^{Q}}{d \omega^{Q}} H_{1}(\omega)\right|_{\omega=0}=\left.\frac{d^{Q}}{d \omega^{Q}} H_{d}\left(e^{j \omega}\right)\right|_{\omega=0}
$$

for $Q=0,1, \ldots, 2 M-1$. These conditions can be further simplified to two sets of linear equations with unknowns $\left\{a_{i}^{(1)} \mid i=0,1, \ldots, M-1\right\}$ and $\left\{b_{i}^{(1)} \mid i=0,1, \ldots, M-1\right\}$. These two subsets of equations are

$$
\sum_{i=0}^{M A-1} a_{i}^{(1)}(2 i)^{Q}=d^{Q} ; \quad Q=0,2, \ldots, 2 M-2
$$

$$
\text { and }
$$

$$
\sum_{i=0}^{M-1} b_{i}^{(1)}(2 i+1)^{Q}=d^{Q} ; \quad Q=1,3, \ldots, 2 M-1 .
$$

Comparing (13) and (14) with (5) and (6), we obtain a closed-form solution of $a_{i}^{(1)}=A_{i}(d)$ and $b_{i}^{(1)}=d B_{i}(d) /(2 i+1)$. Base on the similar derivation, we can obtain the coefficients of all the 8 types of filters. These solutions are listed in Table 2.

\section{DESIGN EXAMPLES}

In this section, we will study the performance of the proposed filters by 6 design examples. Figs. 1 6 show the magnitude responses and group delays of the proposed filters of type-I, II, III, IV, VI, and VIII, respectively. For each filter, the desired delay $d=0.25$ and $M=2,3,4,5$. The filter orders can be calculated according to Table 1. For example, the orders of type-I filters shown in Fig. 1 are $N=4 M-2=6,10,14,18$. The group delays are normalized by the filter orders. Specifically, the normalized group delay $G(\omega)$ shown in figures is calculated from the original group delay $g(\omega)$ by $G(\omega)=g(\omega)-N / 2$. The type-I filter exhibits a very flat magnitude response over the whole frequency band. In fact, this filter is equivalent to the FIR FD filter proposed in Section IV of [6] since the power series of sine function $\sum p_{n}(-j \sin \omega)^{n}$ can be converted to the type I frequency response in Table 1. Fig 2 shows the magnitude response and group delay of type II filters. The magnitude responses drop from unity at $\omega=\pi / 2$. The magnitude responses of type III filters shown in Fig. 3 fall to zero at $\omega=\pi / 2$ because $H_{3}(\pi / 2)=0$ for any order and delay. The magnitude responses of type IV filters in Fig. 4 also drop at $\omega=\pi / 2$. The frequency responses of filters of type I, II, III, and IV are symmetry about $\omega=\pi / 2$.

The magnitude responses and group delays of type VI and VIII are shown in Figs. 5 and 6 respectively. Type VI and VIII filters are exactly equivalent to the filters proposed in [9], which are not symmetric about $\omega=\pi / 2$ and hence have better frequency responses over the frequency band of $[0, \pi / 2]$ than the type III and IV. Note that the group delay around $\omega=0$ of type VIII is actually -0.25 rather than 0.25 as specified. This is resulted from that we have shifted the impulse response half samples backward to implement the sum of two half-sample delay systems in $H_{8}(z)$.

\section{CONCLUSIONS}

In this article, 8 types of FIR filters are proposed for designing FD systems. By the MF approximation, we solve the coefficients in closed form. Two types of filters are mixed by a full-sample and half-sample systems. It is not realizable in practice to design the filter with such mixture. The other 6 types of filters are given in examples and their magnitude responses and group delays are shown in figures. ind that type IV approximates the desired frequency the mid-band, making these filters are not suitable for 
filtering within the mid-band range. Type I, II, III, VI, and VIII, on the other hand, approximate the desired magnitude and group delay frequency responses well within the lowband range. Our method obtains fractional delay closed form solution. Therefore it will have better flexibility and less design complexity than the conventional least-mean-square (LMS) methods.

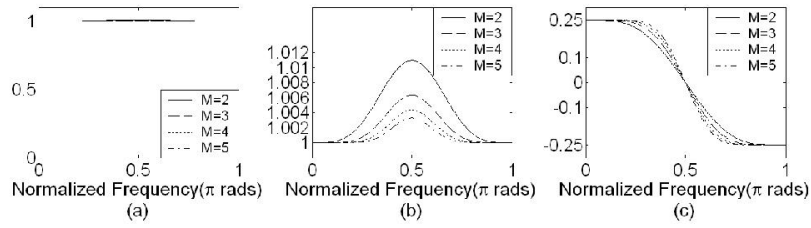

Figure 1. (a) Magnitude responses, (b) magnified magnitude response, and (c) normalized group delays of type-I filters with order $4 M-2, d=0.25$.
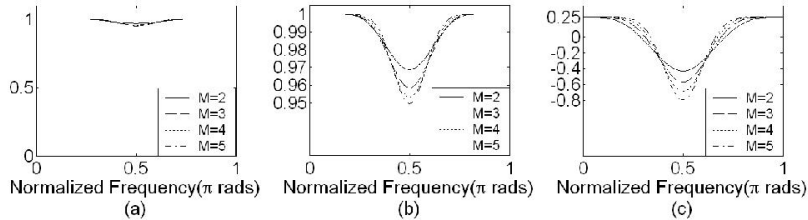

Figure 2. (a) Magnitude responses, (b) magnified magnitude response, and (c) normalized group delays of type-II filters with order $4 M, d=0.25$.

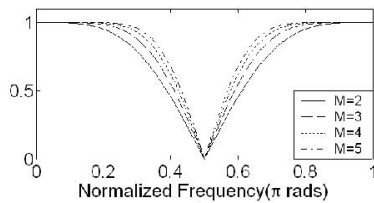

(a)

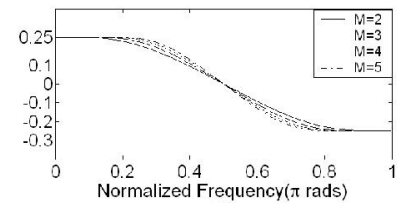

(b)
Figure 3. (a) Magnitude responses and (b) normalized group delays of type-III filters with order $4 M, d=0.25$

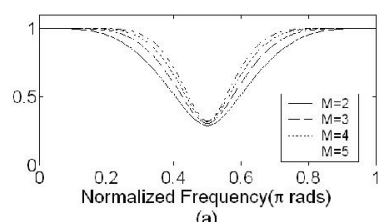

(a)

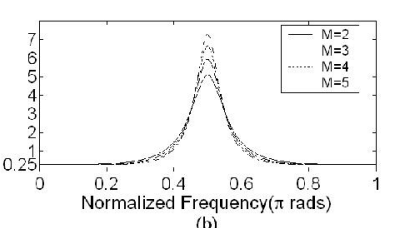

(b)
Figure 4. (a) Magnitude responses and (b) normalized group delays of type-IV filters with order $4 M-2, d=0.25$.

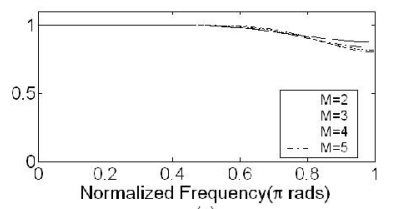

(a)

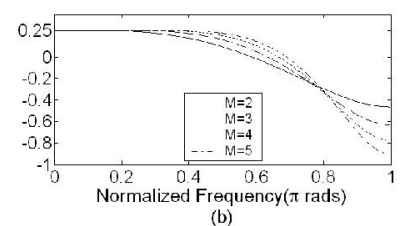

Figure 5. (a) Magnitude responses and (b) normalized group delays of type-VI filters with order $2 M, d=0.25$.
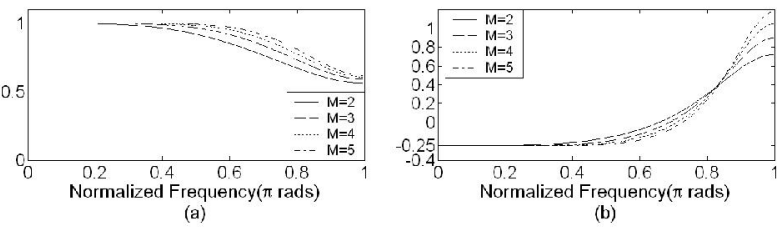

Figure 6. (a) Magnitude responses and (b) normalized group delays of type-VIII filters with order $2 M-1, d=0.25$.

TABLE II. CLOSED-FORM SOLUTIONS OF THE PROPOSED MF FIR FD FILTERS

\begin{tabular}{|c|c|c|c|}
\hline Type & Coefficients & Type & Coefficients \\
\hline $\mathrm{I}[6]$ & $\begin{array}{l}a_{i}^{(1)}=A_{i}(d) \\
b_{i}^{(1)}=d B_{i}(d) /(2 i+1)\end{array}$ & $\mathrm{V}$ & $\begin{array}{l}a_{i}^{(5)}=A_{i}(2 d), \\
b_{i}^{(5)}=2 d B_{i}(2 d) /(2 i+1)\end{array}$ \\
\hline II & $\begin{array}{l}a_{i}^{(2)}=A_{i}(d) \\
b_{i}^{(2)}=d C_{i}(d) /(2 i)\end{array}$ & $\begin{array}{c}\text { VI } \\
{[9]}\end{array}$ & $\begin{array}{l}a_{i}^{(6)}=A_{i}(2 d) \\
b_{i}^{(6)}=d C_{i}(2 d) / i\end{array}$ \\
\hline III & $\begin{array}{l}a_{i}^{(3)}=B_{i}(d) \\
b_{i}^{(3)}=d C_{i}(d) /(2 i)\end{array}$ & VII & $\begin{array}{l}a_{i}^{(7)}=B_{i}(2 d) \\
b_{i}^{(7)}=d C_{i}(2 d) / i\end{array}$ \\
\hline IV & $\begin{array}{l}a_{i}^{(4)}=B_{i}(d), \\
b_{i}^{(4)}=d B_{i}(d) /(2 i+1)\end{array}$ & $\begin{array}{l}\text { VTII } \\
{[9]}\end{array}$ & $\begin{array}{l}a_{i}^{(8)}=B_{i}(2 d), \\
b_{i}^{(8)}=2 d B_{i}(2 d) /(2 i+1)\end{array}$ \\
\hline
\end{tabular}

\section{REFERENCES}

[1] T. I. Laakso, V. Välimäki, M. Karjalainen, and U. K. Laine, "Splitting the unit delay," IEEE Signal Processing Mag., vol. 13, pp. 30-60, Jan. 1996.

[2] A. V. Oppenheim, R. W. Schafer, and J. R. Buck, Discrete-Time Signal Processing, 2nd ed. Englewood Cliffs, NJ: Prentice-Hall, 1999

[3] E. Hermanowicz, "Explicit formulas for weighting coefficients of maximally flat tunable FIR delayers," Electron. Lett., vol. 28, no. 20, pp. 1936-1937, Sept. 1992.

[4] S. Minocha and S. C. Dutta Roy, "A note on the FIR approximation of a fractional sample delay," International Journal of Circuit Theory and Applications, vol. 21 pp. 265-274, 1993.

[5] P. J. Kootsookos and R. C. Williamson, "FIR approximation of fractional sample delay systems," IEEE Trans. Circuits Syst. II, vol. 43, no. 3, Mar. 1996

[6] S. Samadi, M. Omair Ahmad, and M. N. S. Swamy, "Result on maximally flat fractional-delay systems," IEEE Trans. Circuits Syst. I, vol. 51, no. 11, Nov. 2004.

[7] S. C. Pei and P. H. Wang "Closed-form design of maximally flat FIR Hilbert transformers, differentiators, and fractional delayers by power series expansion," IEEE Trans. Circuits Syst. I, vol. 48, pp. 389-398, Apr. 2001.

[8] J.-P. Thiran, "Recursive digital filters with maximally flat group delay," IEEE Trans. Circuit Theory, vol. CT-18, pp. 659-664, Nov. 1971.

[9] S. Samadi and H. Iwakura, "Variable Taylor realization and improved approximation of FIR fractional delay systems," Int. J. Circ. Theor. Appl. vol. 26, pp. 513-522, 1998 . 